\title{
DOES THE MINI NUTRITIONAL ASSESSMENT-SHORT FORM PREDICT CLINICAL OUTCOMES IN YOUNGER REHABILITATION PATIENTS?
}

\author{
L. Wegener ${ }^{1}$, S. James ${ }^{1}$, A. Slattery ${ }^{2}$, M. Satanek ${ }^{1,2}$, M. Miller ${ }^{1}$
}

\begin{abstract}
Objectives: To identify the nutritional status of younger patients on admission to rehabilitation using the Mini Nutritional Assessment - Short Form (MNA-SF) and determine whether the MNA-SF has predictive validity for clinical outcomes in this setting. Design: Retrospective case note audit. Setting: Rehabilitation Unit, Repatriation General Hospital, Adelaïde, Australia. Participants: Fifty four patients under 65 years (mean age $52.9 \pm 10$ years, $54 \%$ female). Measurements: Case notes for adults admitted consecutively to rehabilitation were reviewed. Risk of malnutrition was categorised using the MNA-SF. Outcomes measured were length of stay (LOS), complications and poor participation during admission, change in function, discharge to higher level of care, and acute readmissions and mortality 18 months post discharge. Results: Fourteen (26\%) subjects were malnourished and $28(52 \%)$ were at risk of malnutrition as classified by the MNA-SF. There were no significant differences in clinical outcomes between patients classified as malnourished or at risk of malnutrition and those of normal nutritional status. Conclusion: Over three quarters of subjects were classified as malnourished or at risk of malnutrition. These patients were more likely to have adverse clinical outcomes than their well-nourished counterparts but the difference was not significant. Further research is required to investigate the validity of the MNA-SF and other nutrition screening and assessment tools for adults under 65 years old undergoing rehabilitation.
\end{abstract}

Key words: Nutritional status, malnutrition screening, outcomes, rehabilitation, younger adults.

\section{Introduction}

Malnutrition is common amongst adults undergoing rehabilitation, affecting an estimated 30 to $50 \%$ of patients (1). During hospitalisation, multiple factors contribute to malnutrition, including inadequate nutritional intake, increased nutritional requirements, poor absorption and nutrient losses (2). Patients undergoing rehabilitation are predominantly transferred directly from the acute care setting and are therefore more likely to be poorly nourished on admission to rehabilitation. As in the acute care setting, malnutrition is commonly overlooked in the rehabilitation setting, often leading to further deterioration of nutritional status (1).

The impact of diminishing nutritional status for these patients is significant. Malnutrition is an important predictor of morbidity and mortality and, in the

1. Flinders University, Department of Nutrition and Dietetics, Adelaide, South Australia; 2. Repatriation General Hospital, Department of Nutrition and Dietetics, Adelaide, South Australia

Corresponding Author: Michelle Miller, Department of Nutrition \& Dietetics, Flinders University, GPO Box 2100, Adelaide SA 5001, Ph: +61 88204 5328, Fax: +61 88204 6406, Email: michelle.miller@flinders.edu.au

Received July 17, 2013

Accepted for publication May 5, 2014 rehabilitation setting in particular, it has been associated with prolonged length of stay (LOS), poorer discharge outcomes, poorer function, participation and quality of life (3-6). It is therefore important to identify and treat malnutrition as early as possible during the rehabilitation admission.

According to the Dietitians Association of Australia endorsed evidence based practice guidelines for the nutritional management of malnutrition in adult patients, there are two screening tools recommended for use in the rehabilitation setting, the Mini Nutritional Assessment Short Form (MNA-SF) and the Rapid Screen (1). The MNA-SF is a sensitive, quick, non-invasive nutrition screening tool which incorporates six out of the 18 items from the Mini Nutritional Assessment (MNA). It can be administered with minimal training and has been validated for older adults in a diverse range of settings including acute care, residential care, the community and rehabilitation (7-11). The MNA-SF was revised in 2009 so that a 'malnourished' category could be identified in addition to 'normal nutritional status' and 'at risk of malnutrition' (12). The Rapid Screen, developed in South Australia, comprises two items, body mass index and 
weight loss, and has been validated for adults aged over 65 years (4).

Chronic diseases such as diabetes, some cancers, cardiovascular disease, sleep apnoea and hypertension are being diagnosed in adults at increasingly younger ages due to an increase in the prevalence of overweight and obesity in this age group $(13,14)$. A rise in the number of younger adults admitted to acute care as a result of such diseases could be expected in future years, with a proportion of these likely to require inpatient rehabilitation services. It is speculated that while these patients may be admitted to the acute care setting well nourished, they may be exposed to the same risks that predispose the elderly to deterioration in nutritional status throughout their admission, ultimately arriving in rehabilitation with a sub-optimal nutritional status. It is hence important to be able to identify these at risk patients rapidly to ensure early implementation of nutrition interventions that will support their recovery. Currently however, there is no malnutrition screening tool validated for use in adults under 65 years old in the rehabilitation setting.

Given the absence of a validated screening tool for younger rehabilitation patients and the fact that the MNA-SF is so widely used across different settings including rehabilitation, it would be beneficial if the same tool could be applied to this population of younger rehabilitation patients. Therefore it would be of interest to see if the MNA-SF has any predictive value for clinical outcomes in rehabilitation for this younger age group. An examination of the manner in which younger adults respond to the items of the MNA-SF as compared with older adults, for whom the tool has been validated, would also be useful.

This study therefore aims to identify the nutritional status of younger patients on admission to rehabilitation using the MNA-SF and to determine whether the MNASF has predictive validity for clinical outcomes in this age group. In particular, this study aims to investigate the tool's predictive validity for LOS, change in level of care on discharge, change in function during rehabilitation admission, complications during rehabilitation stay, poor participation, unplanned readmission to hospital and mortality at eighteen months post discharge. Additionally, the study aims to compare how younger adults and older adults respond to the items of the MNASF.

\section{Methods}

Data was collected as part of a retrospective case note audit conducted at the Repatriation General Hospital $(\mathrm{RGH})$. The RGH is a university affiliated teaching hospital with a rehabilitation unit consisting of three wards which accommodate a total of 55 patients. Case notes for all adult patients admitted consecutively to the hospital's Rehabilitation Unit between 6 April 2010 and 15 November 2010 were examined.

The study was approved by the Southern Adelaïde Clinical Human Research Ethics Committee. Patient consent was not required as all the information collected from case notes formed the basis of routine quality assurance audits and was de-identified.

Gender, age, diagnosis and Mini Mental State Examination (MMSE) (15) results on admission were collected from the case notes after discharge from the rehabilitation ward. Diagnosis was categorised into three groups: neurological, orthopaedic and other, which included functional decline, vascular and gastrointestinal surgery.

\section{Nutritional assessment}

The MNA-SF comprises six multiple choice questions. Item A relates to whether food intake has declined, including a grading of the severity of appetite loss. Item B relates to whether the patient's weight has decreased over the last three months, with a choice of four options: weight loss greater than three kilograms; does not know; weight loss of one to three kilograms; or no weight loss. Question C involves a rating of the patient's mobility as either bed or chair-bound; able to get out of a chair or bed but not able to go out; or able to go out. Item $\mathrm{D}$ pertains to the patient's experience of psychological stress or acute disease in the last three months and Question E relates to whether the patient has neuropsychological problems, categorised as severe dementia or depression; mild dementia; or no neuropsychological problems. The last question involves categorising the patient's Body Mass Index (BMI).

The MNA-SF was administered by a ward dietitian within 48 hours of admission to the rehabilitation unit. If the patient was unable to answer any of the first five questions, the patient's nurse or family member was consulted or medical records checked as recommended in the guidelines for the administration of the MNA-SF (16).

In order to calculate BMI, weight was measured to the nearest $0.01 \mathrm{~kg}$ in light clothing without shoes, using a calibrated weigh chair (A\&D FV 150K) and was taken by a rehabilitation nurse on admission to the ward. Ulna length was measured by the dietitian during administration of the MNA-SF and was used to estimate height. This has been shown to predict height accurately in a wide range of patients $(17,18)$. Estimated BMI $(\mathrm{kg} / \mathrm{m} 2)$ was then calculated using admission weight and height.

The MNA-SF score was used to classify patients as 'normal nutritional status', 'at risk of malnutrition' and 'malnourished' as per the MNA-SF guidelines (16). Referral and provision of nutrition support during admission was also documented from medical records. 


\section{Measurement of clinical outcomes}

LOS and admission to a higher level of care were determined from case notes after discharge from the rehabilitation ward. Change in function during rehabilitation admission was calculated using admission and discharge FIM $^{\mathrm{TM}}$ scores. The $\mathrm{FIM}^{\mathrm{TM}}$ is a measure of severity of disability and is widely accepted for use in rehabilitation (19). It comprises thirteen items relating to disability in motor functions and five items relating to disability in cognitive functions. Possible scores range from $18-126$ and a higher score indicates greater independence. FIM ${ }^{\mathrm{TM}}$ scores were generated from assessments made by the ward physiotherapists.

Incidence of complications such as urinary tract infections, respiratory infections, new and/or deterioration in wounds or falls during admission were gleaned from nursing and medical officer case note entries after discharge. Poor participation was defined as the patient not participating in rehabilitation activities on more than one occasion. This was determined from medical and allied health case note entries after the patients' discharge from rehabilitation. Readmissions and deaths were recorded from the hospital admissions software 'OACIS' (Open Architecture Clinical Information System) eighteen months after discharge from rehabilitation. Readmissions were counted if they involved an overnight stay.

\section{Statistical Analysis}

Patient admission characteristics for both younger and older patients were summarised using descriptive statistics, mean (SD) or median (IQR) according to data distribution as well as number of patients and percentages. The Mann-Whitney U test was used to compare continuous characteristics such as BMI and FIM $^{\mathrm{TM}}$ between younger and older patients.

The results of the MNA-SF between the age groups were investigated in terms of the subsequent categories as well as the individual items of the survey.

Clinical outcomes for patients under 65 years of age were compared to the MNA-SF results. Consistent with previous work and the relative small sample size, patients who were classified as malnourished (MNA-SF $<8)(n=14)$ were grouped together with those classified as at risk of malnutrition (MNA-SF $>8$ ) for statistical analyses $(n=42)$.

The chi-square test was used for categorical characteristics such as poor participation and death 18 months post discharge. The Fishers exact test was used when numbers in each group were insufficient for the Chi-squared test. For continuous characteristics such as change in FIM and LOS the Kruskal-Wallis test was applied.

Table 1

Patient characteristics on admission to rehabilitation according to age group [all results expressed as n (\%) unless otherwise stated]

\begin{tabular}{|c|c|c|c|}
\hline Characteristic & $<65$ years old $(n=54)$ & $\geq 65$ years old $(n=181)$ & $\overline{\mathbf{P}}$ \\
\hline Mean age \pm SD (years) & $53 \pm 10$ & $81 \pm 7$ & 0.000 \\
\hline Female & $29(54)$ & $104(58)$ & 0.601 \\
\hline Usual residence & & & 0.603 \\
\hline Community & $50(98)$ & $163(96)$ & \\
\hline HLC & $1(2)$ & $4(2)$ & \\
\hline LLC & $0(0)$ & $3(2)$ & \\
\hline Diagnosis category & & & 0.542 \\
\hline Neurological $^{*}$ & $28(54)$ & $92(52)$ & \\
\hline Orthopaedict & $16(31)$ & $66(38)$ & \\
\hline Otherf & $8(15)$ & $18(10)$ & \\
\hline MNA-SF score (0-14) (mean \pm SD) & $9.3 \pm 2.5$ & $9.3 \pm 2.3$ & 0.748 \\
\hline MNA-SF category & & & 0.939 \\
\hline Normal nutritional status & $12(22)$ & $39(22)$ & \\
\hline At risk of malnutrition & $28(52)$ & $98(54)$ & \\
\hline Malnourished & $14(26)$ & $43(24)$ & \\
\hline Body mass index $\left(\mathrm{kg} / \mathrm{m}^{2}\right)$ (median [IQR]) & $27.6(24.3,31.3)$ & $25.5(23.2,29.6)$ & 0.045 \\
\hline MMSE score $(0-30)($ mean \pm SD $) \S$ & $27 \pm 4.8$ & $24 \pm 5$ & 0.000 \\
\hline FIM (TM) score (18-126) (median [IQR])I & $91(77,104)$ & $85(69,94)$ & 0.010 \\
\hline
\end{tabular}

*Includes stroke, diagnosis related to the spine, neurological diseases, neurosurgery \& subdural haematoma; +Includes fractured neck or femur, knee replacement, hip replacement, fractured spine \& multiple fractures; łIncludes functional decline, vascular \& gastrointestinal surgery; §Higher score indicates better cognitive function; ФHigher score indicates better functional status 


\section{Results}

Two hundred and thirty-seven patients were admitted to rehabilitation at the RGH in the period between 6 April 2010 and 15 November 2010. Approximately one quarter of these admissions were under 65 years of age. The basic admission characteristics of both the younger and older patients are summarised in Table 1.

\section{Mini Nutritional Assessment - Short Form (MNA-SF)}

For the patients under 65 years of age the MNA-SF classified $26 \%(\mathrm{n}=14)$ as malnourished and $52 \%(\mathrm{n}=28)$ as at risk of malnutrition on admission to rehabilitation. Thus, over three quarters of patients under 65 years old admitted to rehabilitation were classified as either malnourished or at risk of malnutrition.

The results of the individual items of the MNA-SF between the age groups are detailed in table 2. The response to the question regarding recent weight loss was significantly different between younger and older patients $\left(X^{2} 9.165, P=0.027\right)$. Younger patients were more likely to know whether they had lost weight and therefore respond with an option other than 'do not know' compared to older patients. There was no significant difference between the answers for the other items.

The 181 patients who were 65 years or older were excluded from further analysis and are reported on separately in Slattery et al (20). There were 54 patients remaining in the study after two patients were excluded due to extreme FIM $^{\mathrm{TM}}$ values (both received the lowest score of 18). For the remaining sample, mean (SD) age was $52.9( \pm 10)$ years, $30(54 \%)$ patients were female and the mean (SD) MMSE was $27 \pm 4.8$. Nearly all patients (98\%) lived at home prior to admission.

Patients who were classed as of normal nutritional status had slightly higher admission FIM ${ }^{\mathrm{TM}}$ than patients who were malnourished or at risk of malnutrition $[\mathrm{Md}=$ 91 (IQR 86, 107) and Md = 99 (IQR 75, 102), respectively], however this difference was not statistically significant $\left(X^{2} 1.220, P=0.269\right)$.

There were no significant associations between MNASF category and diagnostic category $\left(\mathrm{X}^{2} 0.596 \mathrm{P}=0.817\right)$.

\section{Clinical outcomes}

Clinical outcomes of patients according to the two aggregated MNA-SF categories are shown in table 3. There were no significant differences in clinical outcomes according the MNA-SF category.

Less than a third of patients $(\mathrm{n}=14)$ experienced one or more complications during their rehabilitation admission. When comparing these results between the two MNA-SF

Table 2

Response to MNA-SF items according to age group [all values reported as n (\%)] (calf circumference not included)

\begin{tabular}{|c|c|c|c|}
\hline MNA-SF Item & $<65$ years $(n=54)$ & $\geq 65$ years $(n=181)$ & $\bar{P}$ \\
\hline A. Food intake declined & & & $0.385^{*}$ \\
\hline Severe decrease & $4(7)$ & $11(6)$ & \\
\hline Moderate decrease & $21(29)$ & $73(42)$ & \\
\hline No decrease & $28(52)$ & $90(52)$ & \\
\hline B. Involuntary weight loss in last 3 months & & & $0.027^{*}$ \\
\hline$>3 \mathrm{~kg}$ & $16(30)$ & $35(20)$ & \\
\hline Does not know & $2(4)$ & $35(20)$ & \\
\hline 1 to $<3 \mathrm{~kg}$ & $7(13)$ & $25(14)$ & \\
\hline No weight loss & $29(54)$ & $79(45)$ & \\
\hline C. Mobility & & & $0.065^{*}$ \\
\hline Bed or chair bound & $9(17)$ & $17(10)$ & \\
\hline Able to get out of bed/ chair but does not go out & $18(33)$ & $60(35)$ & \\
\hline Goes out & $25(46)$ & $97(56)$ & \\
\hline D. Psychological stress or acute disease in past 3 months & $51(94)$ & $165(94)$ & $0.965^{*}$ \\
\hline E. Neuropsychological problems & & & $0.113+$ \\
\hline Severe dementia or depression & $4(2)$ & $5(2)$ & \\
\hline Mild dementia & $1(0.5)$ & $2(1)$ & \\
\hline No psychological problems & $48(21)$ & $166(73)$ & \\
\hline F. BMI & & & $0.145^{*}$ \\
\hline$<19$ & $6(12)$ & $11(7)$ & \\
\hline 19 to $<21$ & $0(0)$ & $12(7)$ & \\
\hline 21 to $<23$ & $9(17)$ & $23(14)$ & \\
\hline$\geq 23$ & $37(71)$ & $123(73)$ & \\
\hline
\end{tabular}

${ }^{*}$ Chi-squared test for independence; +Chi-squared test for independence with Fishers exact 
Table 3

Clinical outcomes of patients according to MNA-SF category (combining groups at risk of malnutrition and malnourished) [all values reported as $\mathrm{n}(\%)$ unless otherwise specified] $(\mathrm{n}=54)$

\begin{tabular}{|c|c|c|c|}
\hline & $\begin{array}{l}\text { Normal nutritional } \\
\text { status }(12-14)(n=12)\end{array}$ & $\begin{array}{l}\text { At risk of malnutrition } \\
\text { or malnourished }(0-11)(n=42)\end{array}$ & $\bar{P}$ \\
\hline$\geq 1$ complication during admission & $2(4)$ & $12(24)$ & $0.471^{*}$ \\
\hline Poor participation & $2(4)$ & $6(12)$ & $1.00+$ \\
\hline Deceased at 18 months post discharge & $0(0)$ & $6(11)$ & $0.319 \neq$ \\
\hline Length of stay in rehabilitation (days) [median (IQR)] & $16(8,32)$ & $19(14,35)$ & $0.303 \S$ \\
\hline Discharged to a higher level of care & $2(4)$ & $5(10)$ & $0.656 \rrbracket$ \\
\hline Change in FIM [median (IQR)] & $14(7,20)$ & $17(11,28)$ & $0.269 \S$ \\
\hline Acute readmissions 18 months post discharge & $4(33)$ & $19(45)$ & $0.525^{*}$ \\
\hline Acute readmissions 18 months post discharge [median (IQR)] & $2.5(1,5)$ & $1(1,2)$ & $0.460 \S$ \\
\hline
\end{tabular}

${ }^{*}$ Chi-squared test for independence; + Chi -squared test for independence with Fishers exact; $¥$ Chi- squared test for independence; §Kruskal-Wallis test; $\mathbb{I}$ Chi-squared test for independence with Fishers exact

categories there were no significant differences $\left(X^{2} 0.916\right.$, $\mathrm{P}=0.471$ ).

More patients in the malnourished/at risk of malnutrition group were considered poor participators during their admission $(n=6)$ compared to those of normal nutritional status $(n=2)$. However this difference did not reach statistical significance $\left(X^{2} 0.020, P=1.00\right)$.

Median LOS was longer for patients classified as malnourished or at risk of malnutrition (Md 19 (IQR 14, 35) than for those with normal nutritional status (Md 16 (IQR 8,32 ) but the difference was not statistically significant $(P=0.303)$.

Discharge to a higher level of care was an uncommon occurrence with only 7 cases reported. There were $4 \%$ $(n=2)$ and $10 \%(n=5)$ of cases for those classified by the MNA-SF as normal nutritional status, and at risk of malnutrition/malnourished respectively. However these differences were not statistically significant $\left(\mathrm{X}^{2} 0.138, \mathrm{P}=\right.$ $0.656)$.

Acute admissions 18 months post discharge were more likely for patients in the at risk of malnutrition/malnourished group [45\% ( $n=19)]$ compared to the normal nutritional status group [33\% (n =4)] but this was not statistically significant $\left(\mathrm{X}^{2} 0.451, \mathrm{P}\right.$ $=0.525)$.

Death within 18 months after rehabilitation discharge was also uncommon $(n=6)$ and only occurred in patients who were classified as malnourished or at risk of malnutrition. However, comparisons between the groups were not statistically significant $\left(X^{2} 1.929, P=0.319\right)$.

\section{Discussion}

This study explored the predictive validity of the MNA-SF for relevant outcomes in younger rehabilitation patients. Malnutrition and risk of malnutrition as classifed by the MNA-SF were common in this group. Younger adults responded to the questions of the MNA-
SF similarly to older adults except for the item pertaining to recent weight loss. A trend was observed for patients classified as malnourished or at risk of malnutrition to have poorer clinical outcomes than those of normal nutritional status, however these differences were not statistically significant.

The incidence of malnutrition and risk of malnutrition as classified by the MNA-SF was identical to that of the older adults admitted to RGH over the same period of time, with $78 \%$ of both patient groups assessed as malnourished or at risk of malnutrition (20). This was comparable to findings of other studies investigating rates of malnutrition in older rehabilitation patients, such as Charlton et al's study of 2076 Australian rehabilitation patients, of whom $84.5 \%$ were assessed as malnourished or at risk of malnutrition using the full MNA (21). Similarly, Compan et al identified $87 \%$ of a sample of 196 patients as malnourished or at risk of malnutrition using the MNA and Kaiser et al's more recent study found $86.7 \%$ of 99 rehabilitation patients assessed using the MNA were at risk or malnourished $(22,9)$. Other studies examining adults of all ages undergoing rehabilitation have identified $49 \%$ of patients as malnourished using the Subjective Global Assessment (SGA), a much higher proportion than the $26 \%$ assessed as malnourished in this study (23). However the proportion of patients at risk of malnutrition is not measured by the SGA and thus was not reported.

Patients who were classified as malnourished or at risk of malnutrition using the MNA-SF were not found to be at significantly higher risk of selected adverse outcomes in this study. This is unlike the outcomes for older adults admitted to the same facility over the same time period. Those patients who were at risk of malnutrition or malnourished in the older group had longer LOS and were less likely to participate consistently in rehabilitation activities (20).

The lack of a significant association between nutritional status and outcomes is also in contrast to 
findings of other authors. Charlton et al found that LOS was higher in older adults who were malnourished and at risk of malnutrition while Visvanathan et al found that malnourished rehabilitation patients were more likely to be discharged to a higher level of care and Neumann et al found that older rehabilitation patients at risk or malnourished according to the MNA had longer LOS, poorer function and quality of life and had more chance of being discharged to a higher level of care $(21,4,3)$. All of these studies have examined outcomes for older adults, however very few studies have investigated nutritional status and its bearing on rehabilitation outcomes for younger groups. Nip et al studied outcomes for a sample of 100 stroke patients of mean (SD) age 69 (15) years, and demonstrated that higher energy intake early in the rehabilitation admission predicted greater rehabilitation gain, but did not find a relationship with nutritional status (measured using the MNA) as such (6).

The lack of predictive validity of the MNA-SF found in this study may be attributed to the fact that the sample size was too small to show a relationship between nutritional status and outcomes. This is plausible given that there was a trend towards patients classified as malnourished or at risk of malnutrition experiencing all adverse outcomes measured more frequently than their well-nourished counterparts. The number of adverse events actually recorded for this age group was also small compared to those experienced by older adults admitted over the same time period, making it difficult to measure any association with nutritional status. A study involving a larger sample size and perhaps a longer follow up may establish statistically significant associations between MNA-SF category and clinical outcomes in this age group. Additionally, there may be more age-appropriate outcomes with which an association would be more evident.

Alternatively, it is possible that the lack of a significant association between MNA-SF category and clinical outcomes may be due to the fact that the MNA-SF is simply not appropriate for use in younger adults. Although there was no significant difference in the way that five of the six MNA-SF items were answered by the younger adults compared with older adults, it was evident that the two age groups answered differently for the MNA-SF question pertaining to weight loss. This appears to be due to the fact that a larger proportion $(20 \%)$ of older adults did not know if they had lost weight compared with only $3.7 \%$ of the younger adults. This therefore affected the way that the item was scored and may have impacted on the efficacy of the tool.

The MNA-SF also differs to screening tools validated in both younger and older adults, such as the Malnutrition Universal Screening Tool (MUST) (24) and the Simplified Nutritional Assessment Questionnaire (SNAQC) (25) in that it includes three key items relating to the presence of psychological stress, mobility and neuropsychological problems. It is reasonable to speculate that such issues would be common in younger adults given that admission to hospital and rehabilitation is likely to cause at least some level of stress and that mobility is likely to be impaired for patients who have suffered lengthy acute hospital admissions or other conditions requiring rehabilitation, regardless of age. In fact, this is reflected by the similarity in how younger and older adults answered these questions. It was noted that in both age groups the majority of patients reported to have decreased mobility and psychological stress or acute disease. However, the impact of these factors on nutritional status may not be as profound in younger adults, thereby interfering with the performance of the MNA-SF in younger adults.

Hence further research may be required to explore alternatives for malnutrition screening tools for younger adults in rehabilitation. A larger study of the MNA-SF may establish predictive validity for clinical outcomes in this age group or the MNA-SF may need to be refined, with minor changes to the item relating to weight loss, potentially making it more applicable for this age group. Alternatively, the efficacy of other nutrition screening tools for younger adults, such as the Rapid Screen, or a tool validated in the acute care setting such as the MUST could be validated for both young and old in rehabilitation.

The advantage of this study was that it employed a consecutive recruitment method and had a high response rate $(96 \%)$, making the study sample more representative. Additionally, the MNA-SF was administered by only two dietitians, thus limiting inter-observer variation in the screening process. However, there were some limitations which need to be taken into account. The group of younger adults in this study may not be representative of younger rehabilitation patients in general, due to the fact that this particular facility admits very few spinal and severe trauma patients compared with some other major rehabilitation facilities. Readmissions to acute care were only collected from public hospital records, so some readmissions may not have been captured and mortality data was only taken from OACIS which does not provide a comprehensive record of deaths. Due to the nonexperimental design of the study patients who were assessed as malnourished or at risk of malnutrition received nutrition intervention. Therefore the lack of significant associations between malnutrition and clinical outcomes could be attributed to improvements in nutritional status due to nutrition intervention during admission. The validity testing performed was also only addressing predictive validity, a comparison to a reference standard was not included. Finally, as discussed above, the sample size in this study was relatively small, as was the total number of adverse events. Future research directions might include a larger study to avoid risk of type 2 error, inclusion of an 
objective and comprehensive assessment of nutritional status to be used as a reference standard and if our findings are confirmed, refinement of the MNA-SF to address deficits and improve ability to be used across the entirety of patients admitted to the rehabilitation setting.

In conclusion, malnutrition is common in the rehabilitation setting amongst younger adults and although validated screening tools are available for its identification in older adults undergoing rehabilitation, there is no such instrument currently validated for younger adults in this setting. Ideally the same tool would be used across all age groups in the rehabilitation setting for efficiency purposes, however this study could not demonstrate that the MNA-SF has predictive validity for relevant clinical outcomes in younger adults. Further research into the appropriateness of the tools currently validated for rehabilitation or alternatively, investigation of the validity of other nutrition screening tools in the rehabilitation setting is required.

Acknowledgements: The authors would like to thank the Nutrition \& Dietetics Department of Flinders University for providing financial support for the initial stages of the case note audit. We are also grateful to Karen Storah for her work in data collection.

Conflicts of interest: The authors have no conflicts of interest to disclose.

\section{References}

1. Watterson C, Fraser A, Banks M et al. Evidence based practice guidelines for the nutritional management of malnutrition in adult patients across the continuum of care. Nutr Diet 2009;66:1-34

2. Barker L, Gout B, Crowe T. Hospital malnutrition: prevalence, identification and impact on patients and the healthcare system. Int J Env Res Pub Health 2011;8:514-527.

3. Neumann S, Miller M, Daniels L, Crotty M. Nutritional status and clinical outcomes of older patients in rehabilitation. J Hum Nutr Diet 2005;18:129-136.

4. Visvanathan R, Penhall R, Chapman I. Nutritional screening of older people in a sub-acute care facility in Australia and its relation to discharge outcomes. Age \& Ageing 2004;33:260-265.

5. Kaur S, Miller M, Halbert J, Giles L, Crotty M. Nutritional status of adults participating in ambulatory rehabilitation. Asia Pac J Clin Nutr 2008;17:199 207.

6. Nip W, Perry L, McLaren S, Mackenzie A. Dietary intake, nutritional status and rehabilitation outcomes of stroke patients in hospital. J Hum Nutr Diet 2011;24:460-469.

7. Ranhoff A, Gjoen A, Mowe M. Screening for malnutrition in elderly acute patients: the usefulness of the MNA. J Nutr Health Aging 2005;9:221-225.

8. Rubenstein L, Harker I, Salva A et al. Screening for undernutrition in geriatric practice: developing the short-form Mini-Nutritional Assessment (MNA-SF). J Gerontol A Biol Sci Med Sci 2001;56:M366-M372.

9. Kaiser MJ, Bauer JM, Uter W et al. Prospective validation of the modified Mini Nutritional Assessment Short-Forms in the community, nursing home and rehabilitation setting. JAGS 2011;59:2124-2128.

10. Phillips MB, Foley AL, Barnard R, Isenring EA, Miller MD. Nutritional screening in community-dwelling older adults: a systematic literature review. Asia Pac J Clin Nutr 2010;19:440-9.

11. Neumann SA, Miller MD, Daniels LA, Ahern M, Crotty M. Inter-rater reliability and validity of the Mini Nutritional Assessment in older Australians undergoing rehabilitation. Nutr Diet 2007;64:179-85.

12. Kaiser M, Bauer J, Ramsch $C$ et al. Validation of the Mini Nutritional Assessment Short- Form: A practical tool for identification of nutritional status. J Nutr Health Aging 2009;13:782-788.

13. National Preventative Health Taskforce Obesity Working Group (2009) Australia: the healthiest country by 2020 . Technical Report No 1 . Obesity in Australia: a need for urgent action. Commonwealth of Australia. http:/ / www.health.gov.au/internet/ preventativehealth/publishing.nsf/Co ntent/tech-obesity. Accessed 10 May 2013.

14. Australian Bureau of Statistics. National Health Survey 2004-05: Summary of results. ABS cat.no. 4364.0. Canberra:ABS, available at: http://www.abs. gov.au/ Accessed 03 September 2013

15. Folstein MF, Folstein SE, McHugh PR. Mini-mental state. A practical method for grading the cognitive state of patients for the clinician. J Psychiatr Res 1975;12:189-198.

16. Nestle Nutrition Institute (2009) Mini Nutritional Assessment. http:/ / www.mna-elderly.com/forms/mini/mna_mini_english.pdf. Accessed 15 December 2012.

17. Auyeung TW, Lee JSW, Kwok T, Leung J, Leung PC \& Woo J. Estimation of stature by measuring fibula and ulna bone length in 2443 older adults. J Nutr Health Aging 2009;13: 931-936.

18. Barbosa VM, Stratton RJ, Lafuente E, Elia M. Ulna length to predict height in English and Portuguese patient populations. Eur J Clin Nutr 2012;66:209-15.

19. Wright, J (2000) The FIM(TM). The Center for Outcome Measurement in Brain Injury. http:/ / www.tbims.org/combi/ FIM. Accessed 20 April 2013.

20. Slattery A, Wegener L, James S, Satanek M, Miller M. Does the Mini Nutritional Assessment-Short Form predict clinical outcomes at 6 months in older rehabilitation patients? Nutr \& Diet, 2013. Accepted for publication.

21. Charlton K, Nichols C, Bowden S, Lambert K, Barone L, Mason M, Milosavljevic M. Older rehabilitation patients are at high risk of malnutrition: evidence from a large Australian database. J Nut Health Aging 2010;18:622628.

22. Compan B, Di Castri A, Plazi JM, Arnaud-Battandier, F. Epidemiological study of malnutrition in elderly patients in acute, sub-acute and long-term care using the MNA. J Nutr Health Aging 2002;3: 146-151.

23. Beck E, Carrie M, Lambert K, Mason S, Milosavljevic M, Patch C. Implementation of malnutrition screening and assessment by dietitians: malnutrition exists in acute and rehabilitation settings. Aust J Nutr Diet 2001;58:92-97.

24. Elia M. Screening for malnutrition: a multidisciplinary responsibility. Development and use of the Malnutrition Universal Screening Tool ('MUST') for adults. BAPEN, Redditch, 2003.

25. Kruizenga HM, Seidell JC, De Vet HCW, Wierdsma NJ, van Bokhurst-de Van der Schueren MAE. Development and validation of a hospital screening tool for malnutrition: the Short Nutritional Assessment Questionnaire (SNAQ). Clin Nutr 2005;24:75-82. 